\author{
IWONA MAKUCH-PIETRAŚ ${ }^{*}$, NATALIA PIĘTA ${ }^{1}$, MARCIN PIENIAZŻEK $^{2}$ \\ ${ }^{1}$ University of Rzeszow, Department of Agrobiology and Environmental Protection \\ Zelwerowicza 4 Str., 35-601 Rzeszów, Poland \\ ${ }^{2}$ University of Rzeszow, Department of Soil Science, Environmental Chemistry and Hydrology, \\ Zelwerowicza 8 Str., 35-601 Rzeszów, Poland
}

\title{
Impact of recreation and tourism on selected soil characteristics in the Lisia Góra Nature Reserve area (south-east Poland)
}

\begin{abstract}
The aim of the research was to estimate changes in soil properties caused by intensive recreation and tourism in protected areas within the urban borders of Rzeszów (south-east Poland). The research was conducted on two objects: (1) on protected areas of the Lisia Góra Nature Reserve and (2) along the Wisłok Valley. The difference in anthropopressure between the areas was taken into consideration. Soil penetration resistance and moisture were analysed in the spring and autumn period. Soil texture, $\mathrm{pH}$, total carbon, and electrical conductivity (EC) in a 1:10 soil:water suspension were measured in soil material collected in spring. The ion content $\left(\mathrm{Ca}^{2+}, \mathrm{Mg}^{2+}, \mathrm{K}^{+}, \mathrm{Na}^{+}\right)$was measured in the same suspension with an ion chromatograph. There were significant correlations (determined by the Pearson coefficient) between soil acidity, EC, total exchangeable bases, ions of calcium and magnesium, and soil penetration resistance. A significant difference was found in soil penetration resistance between objects (higher in the Wisłok Valley area) and between the distances from the border of the intensively managed area (a decrease as the distance increased). The $\mathrm{Z}$ coefficient (the ratio of sodium ions, the sum of calcium and magnesium ions) was significantly lower for the the Lisia Góra Nature Reserve sites.
\end{abstract}

Keywords: soil compaction, electrical conductivity, "Z" coefficient, tourism, protected area

\section{INTRODUCTION}

The impact of tourism in particular in mountainous protected areas, has been widely discussed in the literature (e.g. Ewertowski and Tomczyk, 2007). However, areas that are the most threatened by tourism and recreation are situated within urban borders (Sikorski et al. 2013). In each valuable natural area, hiking trails have an important role, especially in protected areas, where the aim is protection of nature, development of tourism, and adaptation to exploration by visitors. They serve a variety of ecological (studies of environmental changes, exclusion of protected areas from tourist traffic or control of its intensity) and touristic functions, for example: leisure management, relaxation through the contact with nature, running, cycling, and access to tourist attractions (Bajda-Gołębiewska 2011). Development of many tourist activities is often associated with the growing need for recreation and leads to severe exploitation of the environment and, ultimately, degradation of its quality (BajdaGołębiewska 2011, Machnik 2010, Szpilka and Ziółkowski 2010). The degree of soil environment transformation caused by trampling depends on the character and forms of tourism, proper planning of the development of tourism infrastructure, proper management of tourist traffic, tourists' environmental awareness, and the resistance of the soil to the anthropopressure (Mika 2000). „Soil trampling” is a form of environmental changes leading to an increase in soil density and reduction of air and water properties, which creates unfavourable conditions for the development of soil microorganisms and plants (Mika 2000).

Investigations conducted by many authors indicated that soil exhibited the highest "trampling" level in the direct site of trampling, which however decreased with the distance from that point, regardless of the research period and the sampling site. The density of the soil surface increases surface runoff, leading to the development of erosion processes with effects visible on tourist trails. Therefore, the farther from the trail, the less trampled the soil and vegetation is (Mika 2000, Zdanowicz and Skłodowski 2013).

Therefore, the studies were undertaken to determine how tourism affects the soil environment in the Lisia Góra Reserve and the surrounding recreational used areas in the Wisłok Valley.

\section{MATERIALS AND METHODS}

The study was conducted in the area of the Lisia Góra Reserve and the Wisłok Valley located in the 
southern part of Rzeszów city in Podkarpackie province at an altitude of 200-220 m above sea level. The area of the reserve is 8,1 ha (including 7.40 ha of forest areas; 0.36 ha of roads and 0.35 ha of glades). It is a communal forest, established to ,preserve old-growth oaks with numerous aged specimens for scientific and teaching purposes." A part of the Władysław Szafer natural path is located within the reserve (Mroczek and Kostecka 2008). The use of the area of the Wisłok Valley is very high (even hundreds of pedestrians - date of research 2.08.2009) as shown by the research conducted near the Lisia Góra Reserve (Ćwik 2009). The research conducted in 2008-2010 showed a negative impact of anthropopressure, i.e. flora devastation, ground features changes and negative changes in the landscape. New paths were formed, as shown by the Lisia Góra reserve map (Ćwik and Ćwik 2011). Seven sites with high tourism pressure were selected in the area. Three sites were analysed in the reserve: a bench in the reserve (BR), the first trail in the reserve near the information table (T1) and the second trail in the reserve (T2). Four sites were chosen in the Wisłok Valley: a playground $(\mathrm{P})$, a grill area $(\mathrm{G})$, a trail near a golf pool (TG) and a trail under the bridge (TB). In the spring of 2015, soil samples were collected in every site from a depth of $0-5 \mathrm{~cm}$, from the most stressed part in the area of tourist interference and at a distance of $0.5 \mathrm{~m}$ and $1 \mathrm{~m}$ from every stressed site. In the same period and again in autumn after the end of the tourist season, soil penetration resistance was measured with a pocket piston penetrometer (type 16-TO171) and soil moisture was determined with an Extech hygrometer (model MO750). Soil samples were air-dried, ground, and sieved through a sieve with a diameter of $2 \mathrm{~mm}$. Basic analyses of the physicochemical properties of soils were carried out in the collected soil material. The particle size distribution was determined with the Bouyoucos-Casagrande method modified by Prószyński according to standard PTG 2008, (Polskie Towarzystwo Gleboznawcze 2009). Soil pH was determined potentiometrically in a $1 \mathrm{~mol} \cdot \mathrm{dm}^{-3} \mathrm{KCl}$ solution and distilled water (PN-ISO 10390). Electrical conductivity (EC) was determined conductometrically in an aqueous solution at a 1:10 soil-to-water ratio according to norm PN-ISO 11265+AC1. The content of cations and anions was assessed in the same solution using an ion chromatograph (Breś 2008). The organic matter content was determined with the Tiurin method (Mocek et al. 1997). The content of exchangeable base cations $\left(\mathrm{Ca}^{2+}, \mathrm{Mg}^{2+}, \mathrm{K}^{+}, \mathrm{Na}^{+}\right)$was evaluated after extraction with $1 \mathrm{~mol} \cdot \mathrm{dm}^{-3}$ ammonium acetate at $\mathrm{pH}$ 7 , with the atomic absorption spectrometry technics Hitachi Z-2000 (Ostrowska et al. 2001), and hydrolytic acidity was determined with the Kappen method (Mocek et al. 1997). Salinity index Z, which indicates the relation between the molar ratio of $\mathrm{Na}^{+}$and the sum of $\mathrm{Ca}^{2+}$ and $\mathrm{Mg}^{2+}$ of an aqueous solution at a 1:10 soil-to-water ratio to EC method, is presented (Breś 2008).

The soil material was analysed in duplicate. The results are presented as means and ranges. The Kruskal-Wallis test was used to analyze the differences in soil properties between the sites and distance. Pearson correlation coefficients between physicochemical and chemical soil properties and soil compaction were calculated. All statistical analyses were carried out in the Statistica 12.0 program with a license for the University of Rzeszów.

\section{RESULTS AND DISCUSSION}

The studied soils predominantly exhibited a soil texture of silt loam (mainly the soil from the Lisia Góra reserve) as well as sandy loam and loamy sand, which dominated in the examined sites in the Wisłok Valley. The biggest diversity in terms of grain size composition was noted for the playground, which may be related to the greatest transformations of the area (Table 1).

In spring time, the soil penetration resistance ranged from 2.80 to $5.00 \mathrm{~kg} \cdot \mathrm{cm}^{-2}$. Only the sites from the Lisia Góra reserve (T1 and T2) exhibited significant changes in soil penetration resistance (Table 1). There was a significant downward tendency with the increasing distance from the trail (Table 2). In the autumn period, the soil compaction was in the range of 4.25-5.00 $\mathrm{kg} \cdot \mathrm{cm}^{-2}$, with higher values of soil penetration resistance after the intensive use by pedestrians, in comparison to the spring period. The sites in the Lisia Góra reserve had a significantly higher soil penetration resistance level (Table 1). In contrast to the spring period, there were large differences between the distances, with a significantly decreasing trend along the distance of $1 \mathrm{~m}$ from the trail, which may have resulted from the intensive use of the area by tourists over the holiday period, probably also at the distance of $0.5 \mathrm{~m}$ (Table 2).

Littlemore and Barker (2001), who examined the impact of trampling on forest soils in urban borders reported significantly higher soil compaction in areas that were intensively used by tourists. Zhevelev and Pariente (2008), who compared areas with different pressures confirmed this trend. In their research, Millward et al. (2011) showed a significant influence of vegetation on reducing soil compaction, which can explain the differences in soil compaction between the trails and at a distance of $1 \mathrm{~m}$. In their analysis of the impact of tourism and recreation in protected areas of Au- 
TABLE 1. Selected physicochemical properties of soils from the analysed sites

\begin{tabular}{|c|c|c|c|c|c|c|c|c|}
\hline \multicolumn{2}{|c|}{ Parameter } & $\mathrm{P}$ & G & $\mathrm{TG}$ & $\mathrm{TB}$ & $\mathrm{BR}$ & $\mathrm{T} 1$ & $\mathrm{~T} 2$ \\
\hline & & \multicolumn{7}{|l|}{ Mean range } \\
\hline \multirow[t]{2}{*}{$\overline{\text { SPR }}$} & $\begin{array}{l}\text { spring } \\
\left(\mathrm{kg} \cdot \mathrm{cm}^{-2}\right)\end{array}$ & $\begin{array}{l}4.41 \\
4.10-5.00\end{array}$ & $\begin{array}{l}4.36^{\mathrm{a}} \\
4.20-4.60\end{array}$ & $\begin{array}{l}4.50 \\
4.25-4.80\end{array}$ & $\begin{array}{l}4.66 \\
4.30-5.00\end{array}$ & $\begin{array}{l}4.51 \\
4.25-5.00\end{array}$ & $\begin{array}{l}4.04^{\mathrm{b}} \\
2.80-5.00\end{array}$ & $\begin{array}{l}4.79^{\mathrm{a}, \mathrm{b}} \\
4.25-5.00\end{array}$ \\
\hline & $\begin{array}{l}\text { autumn } \\
\left(\mathrm{kg} \cdot \mathrm{cm}^{-2}\right)\end{array}$ & $\begin{array}{l}4.50^{\mathrm{a}, \mathrm{b}} \\
4.30-4.60\end{array}$ & $\begin{array}{l}4.55^{\text {c.d }} \\
4.00-4.75\end{array}$ & $\begin{array}{l}4.54 \\
4.30-4.60\end{array}$ & $\begin{array}{l}4.73 \\
4.40-5.00\end{array}$ & $\begin{array}{l}4.80^{\mathrm{a}, \mathrm{c}} \\
4.70-5.00\end{array}$ & $\begin{array}{l}4.77^{\text {b.d }} \\
4.50-4.90\end{array}$ & $\begin{array}{l}4.71 \\
4.50-4.80\end{array}$ \\
\hline \multirow[t]{2}{*}{ Moisture } & $\begin{array}{l}\text { spring } \\
(\%)\end{array}$ & $\begin{array}{l}14.09 \\
9.80-17.40\end{array}$ & $\begin{array}{l}14.43 \\
8.30-17.70\end{array}$ & $\begin{array}{l}14.13 \\
8.70-17.60\end{array}$ & $\begin{array}{l}13.25 \\
7.10-17.70\end{array}$ & $\begin{array}{l}12.02 \\
7.00-17.00\end{array}$ & $\begin{array}{l}10.18 \\
5.50-15.30\end{array}$ & $\begin{array}{l}9.76 \\
5.10-15.50\end{array}$ \\
\hline & $\begin{array}{l}\text { autumn } \\
(\%)\end{array}$ & $\begin{array}{l}8.63^{\mathrm{a}, \mathrm{b}} \\
1.80-15.00\end{array}$ & $\begin{array}{l}15.48^{\mathrm{a}, \mathrm{c}} \\
14.10-16.80\end{array}$ & $\begin{array}{l}16.11^{\mathrm{b}, \mathrm{d}} \\
13.20-20.70\end{array}$ & $\begin{array}{l}15.18 \\
9.20-24.00\end{array}$ & $\begin{array}{l}8.11^{\mathrm{c}, \mathrm{de}} \\
1.10-14.00\end{array}$ & $\begin{array}{l}14.55^{\mathrm{e}} \\
4.80-18.90\end{array}$ & $\begin{array}{l}14.37 \\
9.90-17.00\end{array}$ \\
\hline \multicolumn{2}{|c|}{$\begin{array}{l}\text { Fraction }<0.002 \mathrm{~mm} \\
(\%)\end{array}$} & $\begin{array}{l}4.33 \\
2.00-7.00\end{array}$ & $\begin{array}{l}2.33^{\mathrm{a}} \\
1.00-5.00\end{array}$ & $\begin{array}{l}2.53^{\mathrm{b}} \\
0.00-6.00\end{array}$ & $\begin{array}{l}2.60^{\mathrm{c}} \\
1.00-6.00\end{array}$ & $\begin{array}{l}4.47 \\
2.00-7.00\end{array}$ & $\begin{array}{l}3.33 \\
0.00-7.00\end{array}$ & $\begin{array}{l}7.20^{\mathrm{a}, \mathrm{b}, \mathrm{c}} \\
3.00-11.00\end{array}$ \\
\hline \multicolumn{2}{|l|}{$\mathrm{pH} \mathrm{H}_{2} \mathrm{O}$} & $\begin{array}{l}6.34 \\
5.52-7.47\end{array}$ & $\begin{array}{l}6.67^{\mathrm{a}} \\
6.00-7.47\end{array}$ & $\begin{array}{l}6.58^{\mathrm{b}} \\
5.81-7.50\end{array}$ & $\begin{array}{l}6.97^{\mathrm{c}, \mathrm{d}} \\
6.27-7.82\end{array}$ & $\begin{array}{l}4.75^{\mathrm{a}, \mathrm{b}, \mathrm{c}} \\
3.85-6.30\end{array}$ & $\begin{array}{l}5.99 \\
5.20-7.02\end{array}$ & $\begin{array}{l}4.57^{\mathrm{e}, \mathrm{f}} \\
5.00-6.95\end{array}$ \\
\hline \multicolumn{2}{|l|}{$\mathrm{pH} \mathrm{KCl}$} & $\begin{array}{l}5.81^{\mathrm{a}} \\
4.85-6.61\end{array}$ & $\begin{array}{l}5.82^{\mathrm{b}} \\
4.13-6.75\end{array}$ & $\begin{array}{l}6.25^{\mathrm{c}, \mathrm{c}} \\
4.93-7.03\end{array}$ & $\begin{array}{l}6.37^{\mathrm{d}, \mathrm{f}} \\
5.74-7.05\end{array}$ & $\begin{array}{l}3.75^{\mathrm{a}, \mathrm{b}, \mathrm{c}, \mathrm{d}} \\
3.08-5.03\end{array}$ & $\begin{array}{l}5.19 \\
4.28-6.06\end{array}$ & $\begin{array}{l}5.81^{\mathrm{d}} \\
5.00-6.95\end{array}$ \\
\hline \multicolumn{2}{|l|}{$\begin{array}{l}\text { TOC } \\
\mathrm{g} \cdot \mathrm{kg}^{-1}\end{array}$} & $\begin{array}{l}25.87 \\
10.76-40.28\end{array}$ & $\begin{array}{l}36.88^{\mathrm{a}, \mathrm{b}} \\
22.45-53.20\end{array}$ & $\begin{array}{l}19.09^{\mathrm{a}, \mathrm{d}, \mathrm{e}} \\
5.23-27.37\end{array}$ & $\begin{array}{l}26.55 \\
13.84-37.21\end{array}$ & $\begin{array}{l}32.41^{\mathrm{c}, \mathrm{d}} \\
19.37-42.74\end{array}$ & $\begin{array}{l}21.50^{\mathrm{e}} \\
8.30-37.21\end{array}$ & $\begin{array}{l}33.07^{\mathrm{b}, \mathrm{c}} \\
16.61-53.51\end{array}$ \\
\hline \multicolumn{2}{|c|}{$\mathrm{EC}\left(\mu \mathrm{S} \cdot \mathrm{cm}^{-1}\right)$} & $\begin{array}{l}189.30 \\
89.05-534.00\end{array}$ & $\begin{array}{l}216.28 \\
115.25-316.00\end{array}$ & $\begin{array}{l}159.90 \\
121.95-234.00\end{array}$ & $\begin{array}{l}198.86 \\
118.00-256.00\end{array}$ & $\begin{array}{l}96.99 \\
45.701-80.80\end{array}$ & $\begin{array}{l}87.84 \\
53.30-118.55\end{array}$ & $\begin{array}{l}77.33 \\
37.80-165.00\end{array}$ \\
\hline \multicolumn{2}{|c|}{$\mathrm{EH}\left(\mathrm{cmol}(+) \cdot \mathrm{kg}^{-1}\right)$} & $\begin{array}{l}1.08^{\mathrm{a}, \mathrm{b}} \\
0.54-2.18\end{array}$ & $\begin{array}{l}2.03^{c} \\
0.85-4.12\end{array}$ & $\begin{array}{l}0.94^{\mathrm{d}, \mathrm{f}, \mathrm{h}} \\
0.62-1.24\end{array}$ & $\begin{array}{l}1.11^{\mathrm{e}, \mathrm{g}} \\
0.93-1.40\end{array}$ & $\begin{array}{l}11.17^{\mathrm{a}, \mathrm{c}, \mathrm{d}} \\
6.46-22.65\end{array}$ & $\begin{array}{l}4.09^{\mathrm{h}} \\
2.88-5.91\end{array}$ & $\begin{array}{l}7.36^{\mathrm{b}, \mathrm{f}, \mathrm{g}} \\
4.51-13.31\end{array}$ \\
\hline \multicolumn{2}{|c|}{ TEB $\left(\operatorname{cmol}(+) \cdot \mathrm{kg}^{-1}\right)$} & $\begin{array}{l}25.20^{\mathrm{a}} \\
11.96-35.81\end{array}$ & $\begin{array}{l}22.96 \\
19.98-27.38\end{array}$ & $\begin{array}{l}20.04^{\mathrm{b}, \mathrm{d}} \\
21.29-37.11\end{array}$ & $\begin{array}{l}32.84^{\mathrm{c}, \mathrm{e}, \mathrm{f}} \\
22.06-49.61\end{array}$ & $\begin{array}{l}14.62^{\mathrm{a}, \mathrm{b}, \mathrm{c}} \\
12.91-17.36\end{array}$ & $\begin{array}{l}20.21^{\mathrm{f}} \\
14.91-26.12\end{array}$ & $\begin{array}{l}16.41^{\mathrm{d}, \mathrm{c}} \\
9.99-22-63\end{array}$ \\
\hline \multicolumn{2}{|c|}{$\mathrm{CEC}\left(\mathrm{cmol}(+) \cdot \mathrm{kg}^{-1}\right)$} & $\begin{array}{l}25.31^{\mathrm{a}} \\
12.04-35.91\end{array}$ & $\begin{array}{l}23.16 \\
20.27-27.52\end{array}$ & $\begin{array}{l}26.13^{\mathrm{b}, \mathrm{d}} \\
21.40-37.18\end{array}$ & $\begin{array}{l}32.95^{\mathrm{c}, \mathrm{e}, \mathrm{f}} \\
22.17-49.71\end{array}$ & $\begin{array}{l}15.73^{\mathrm{a}, \mathrm{b}, \mathrm{c}} \\
14.11-18.54\end{array}$ & $\begin{array}{l}20.62^{\mathrm{f}} \\
15.41-26.48\end{array}$ & $\begin{array}{l}17.15^{\mathrm{d}, \mathrm{c}} \\
10.84-23.11\end{array}$ \\
\hline \multicolumn{2}{|c|}{$\mathrm{Cl}^{-}\left(\mathrm{mg} \cdot \mathrm{dm}^{-3}\right)$} & $\begin{array}{l}5.54^{\mathrm{a}} \\
1.42-14.95\end{array}$ & $\begin{array}{l}4.68^{\mathrm{b}} \\
1.30-8.98\end{array}$ & $\begin{array}{l}8.30^{\mathrm{d}, \mathrm{f}} \\
1.05-18.75\end{array}$ & $\begin{array}{l}19.42^{\mathrm{c}, \mathrm{e}, \mathrm{g}} \\
4.30-42.38\end{array}$ & $\begin{array}{l}2.62^{\mathrm{c}} \\
1.78-6.32\end{array}$ & $\begin{array}{l}0.78^{\mathrm{a}, \mathrm{b}, \mathrm{f}, \mathrm{g}} \\
0.31-1.44\end{array}$ & $\begin{array}{l}1.25^{\mathrm{d}, \mathrm{e}} \\
0.45-2.38\end{array}$ \\
\hline \multicolumn{2}{|c|}{$\mathrm{Na}^{+}\left(\mathrm{mg} \cdot \mathrm{dm}^{-3}\right)$} & $\begin{array}{l}4.59^{\mathrm{a}, \mathrm{b}} \\
1.03-16.09\end{array}$ & $\begin{array}{l}1.76 \\
0.69-3.05\end{array}$ & $\begin{array}{l}1.93 \\
0.00-5.53\end{array}$ & $\begin{array}{l}15.40^{\mathrm{c}, \mathrm{d}} \\
1.17-34.54\end{array}$ & $\begin{array}{l}1.62 \\
0.44-6.21\end{array}$ & $\begin{array}{l}0.00^{\mathrm{b}, \mathrm{d}} \\
0.00-0.00\end{array}$ & $\begin{array}{l}0.25^{\mathrm{a}, \mathrm{c}} \\
0.00-1.41\end{array}$ \\
\hline \multicolumn{2}{|c|}{$\mathrm{K}^{+}\left(\mathrm{mg} \cdot \mathrm{dm}^{-3}\right)$} & $\begin{array}{l}7.53^{\mathrm{a}, \mathrm{b}} \\
1.90-19.82\end{array}$ & $\begin{array}{l}20.48^{\mathrm{a}, \mathrm{c}} \\
7.80-33.08\end{array}$ & $\begin{array}{l}24.83^{\mathrm{b}, \mathrm{d}} \\
3.28-43.51\end{array}$ & $\begin{array}{l}9.00 \\
5.80-14.39\end{array}$ & $\begin{array}{l}11.26 \\
8.51-15.84\end{array}$ & $\begin{array}{l}12.09 \\
7.84-15.49\end{array}$ & $\begin{array}{l}7.91^{\mathrm{c}, \mathrm{d}} \\
2.97-18.61\end{array}$ \\
\hline \multicolumn{2}{|c|}{$\mathrm{Ca}^{2+}\left(\mathrm{mg} \cdot \mathrm{dm}^{-3}\right)$} & $\begin{array}{l}35.75^{\mathrm{a}, \mathrm{b}, \mathrm{c}} \\
12.54-102.45\end{array}$ & $\begin{array}{l}28.58^{\mathrm{d}} \\
15.01-43.09\end{array}$ & $\begin{array}{l}28.13^{\mathrm{f}} \\
18.89-39.51\end{array}$ & $\begin{array}{l}33.75^{\mathrm{e}, \mathrm{f}, \mathrm{g}} \\
22.51-40.34\end{array}$ & $\begin{array}{l}14.58^{\mathrm{a}, \mathrm{c}} \\
6.56-28.28\end{array}$ & $\begin{array}{l}15.02^{\mathrm{c}, \mathrm{g}} \\
10.42-19.13\end{array}$ & $\begin{array}{l}11.97^{\mathrm{b}, \mathrm{d}, \mathrm{f}} \\
5.47-21.60\end{array}$ \\
\hline \multicolumn{2}{|c|}{$\mathrm{Mg}^{2+}\left(\mathrm{mg} \cdot \mathrm{dm}^{-3}\right)$} & $\begin{array}{l}6.21^{\mathrm{a}, \mathrm{b}, \mathrm{c}} \\
2.47-19.83\end{array}$ & $\begin{array}{l}6.32^{\mathrm{d}, \mathrm{e}, \mathrm{f}} \\
2.99-9.88\end{array}$ & $\begin{array}{l}3.88^{\mathrm{g}} \\
2.19-6.07\end{array}$ & $\begin{array}{l}2.23^{\mathrm{b}, \mathrm{e}} \\
1.65-2.99\end{array}$ & $\begin{array}{l}2.81 \\
1.09-5.29\end{array}$ & $\begin{array}{l}1.41^{\mathrm{c}, \mathrm{f}, \mathrm{g}} \\
0.91-1.90\end{array}$ & $\begin{array}{l}1.98^{\mathrm{a}, \mathrm{d}} \\
0.90-3.73\end{array}$ \\
\hline \multicolumn{2}{|c|}{ "Z" coefficient } & $\begin{array}{l}0.113 \mathrm{a} \\
0.024-0.318\end{array}$ & $\begin{array}{l}0.057 \\
0.013-0.126\end{array}$ & $\begin{array}{l}0.064 \\
0.000-0.189\end{array}$ & $\begin{array}{l}0.421^{c, d} \\
0.038-0.862\end{array}$ & $\begin{array}{l}0.104^{\mathrm{b}} \\
0.027-0.400\end{array}$ & $\begin{array}{l}0.000^{\mathrm{a}, \mathrm{b}, \mathrm{d}} \\
0.000-0.000\end{array}$ & $\begin{array}{l}0.016^{\mathrm{c}} \\
0.000-0.079\end{array}$ \\
\hline \multicolumn{2}{|c|}{ Soil valuation item } & sandy loam & sandy loam & loamy sand & sandy loam & silt loam & silt loam & silt loam \\
\hline
\end{tabular}

Places from Wisłok Valley: P-playground; G-grill area; TG-trail near the golf pool, TB-trail under the bridge; Places from Lisia Góra Reserve: BRbench; T1-1 trail near the information table; T2-2 trail;

a.. Significantly different pairs with $\mathrm{P}<0.05$, SPR - soil penetration resistance, TOC - total organic carbon, EC - electrical conductivity, EH hydrolytic acidity, TEB - total exchangeable bases, CEC-cation exchange capacity, "Z" coefficient - salinity index "Z".

stralia, Pickering and Hill (2007) reported less damage in less frequented sites. Talbot et al. (2003) conducted an experiment that showed increasing soil compaction under the influence of intensive trampling. Similarly, research conducted by Andrés-Abellán et al. (2005) based on significant correlation coefficients showed that greater numbers of tourists contribute to an increase in soil compaction. In forest parks in Poland, Sikorski et al. (2013) showed significant changes in soil compaction under the influence of trampling, affecting the presence of certain plant species. In analysis of soil of an urban park in Hong Kong, Jim (1998) emphasised a significant influence of increased soil compaction in areas with intensive tourism on other soil properties, which has also been presented in this work.

The moisture of the investigated soils in the spring period was estimated at $5.10-17.70 \%$, the results were highly diverse and no trends were distinguished (Table 1). In the autumn period, the soil moisture 
TABLE 2. Selected physicochemical properties of soils at the different distances from every stressed site

\begin{tabular}{|c|c|c|c|c|}
\hline \multicolumn{2}{|c|}{ Parameter } & \multicolumn{3}{|l|}{ Distance (m) } \\
\hline & & 0 & 0.5 & 1 \\
\hline & & \multicolumn{3}{|l|}{ Mean range } \\
\hline \multirow[t]{2}{*}{$\begin{array}{l}\mathrm{SPR} \\
\left(\mathrm{kg} \cdot \mathrm{cm}^{-2}\right)\end{array}$} & spring & $\begin{array}{l}4.69^{\mathrm{a}, \mathrm{b}} \\
4.25-5.00\end{array}$ & $\begin{array}{l}4.43^{\mathrm{a}} \\
3.50-4.90\end{array}$ & $\begin{array}{l}4.27^{\mathrm{b}} \\
4.00-4.80\end{array}$ \\
\hline & autumn & $\begin{array}{l}4.75^{\mathrm{a}} \\
4.40-5.00\end{array}$ & $\begin{array}{l}4.66 \\
4.45-4.90\end{array}$ & $\begin{array}{l}4.56^{\mathrm{a}} \\
4.00-4.80\end{array}$ \\
\hline \multirow[t]{2}{*}{$\begin{array}{l}\text { Moisture } \\
(\%)\end{array}$} & spring & $\begin{array}{l}12.42 \\
5.75-17.70\end{array}$ & $\begin{array}{l}12.43 \\
5.10-17.40\end{array}$ & $\begin{array}{l}12.63 \\
5.50-17.70\end{array}$ \\
\hline & autumn & $\begin{array}{l}11.54^{\mathrm{a}} \\
1.80-17.00\end{array}$ & $\begin{array}{l}13.65 \\
7.10-22.40\end{array}$ & $\begin{array}{l}14.50^{\mathrm{a}} \\
1.10-24.00\end{array}$ \\
\hline \multicolumn{2}{|c|}{$\begin{array}{l}\text { Fraction }<0.002 \\
\mathrm{~mm}(\%)\end{array}$} & $\begin{array}{l}3.56 \\
0.00-10.00\end{array}$ & $\begin{array}{l}3.86 \\
1.00-11.00\end{array}$ & $\begin{array}{l}4.11 \\
1.00-9.00\end{array}$ \\
\hline \multicolumn{2}{|l|}{$\mathrm{pH} \mathrm{H} \mathrm{H}_{2} \mathrm{O}$} & $\begin{array}{l}6.49^{\mathrm{a}} \\
4.25-7.82\end{array}$ & $\begin{array}{l}6.03 \\
4.35-7.51\end{array}$ & $\begin{array}{l}5.97^{\mathrm{a}} \\
3.85-7.59\end{array}$ \\
\hline \multicolumn{2}{|l|}{$\mathrm{pH} \mathrm{KCl}$} & $\begin{array}{l}5.69 \\
3.27-7.03\end{array}$ & $\begin{array}{l}5.30 \\
3.08-7.05\end{array}$ & $\begin{array}{l}5.20 \\
3.47-6.92\end{array}$ \\
\hline \multicolumn{2}{|c|}{ TOC $\left(\mathrm{g} \cdot \mathrm{kg}^{-1}\right)$} & $\begin{array}{l}27.34 \\
10.76-53.50\end{array}$ & $\begin{array}{l}27.18 \\
5.22-53.19\end{array}$ & $\begin{array}{l}29.19 \\
6.45-46.74\end{array}$ \\
\hline \multicolumn{2}{|c|}{$\mathrm{EC}\left(\mu \mathrm{S} \cdot \mathrm{cm}^{-1}\right)$} & $\begin{array}{l}152.77 \\
45.70-534\end{array}$ & $\begin{array}{l}143.60 \\
37.80-298.00\end{array}$ & $\begin{array}{l}140.93 \\
50.70-316.00\end{array}$ \\
\hline \multicolumn{2}{|c|}{$\mathrm{EH}\left(\mathrm{cmol}(+) \cdot \mathrm{kg}^{-1}\right)$} & $\begin{array}{l}3.16 \\
0.54-11.75\end{array}$ & $\begin{array}{l}4.23 \\
0.62-12.84\end{array}$ & $\begin{array}{l}4.52 \\
0.78-22.65\end{array}$ \\
\hline \multicolumn{2}{|c|}{ TEB $\left(\mathrm{cmol}(+) \cdot \mathrm{kg}^{-1}\right)$} & $\begin{array}{l}24.25 \\
11.96-49.61\end{array}$ & $\begin{array}{l}21.28 \\
9.99-36.13\end{array}$ & $\begin{array}{l}22.34 \\
12.71-39.28\end{array}$ \\
\hline \multicolumn{2}{|c|}{$\mathrm{CEC}\left(\mathrm{cmol}(+) \cdot \mathrm{kg}^{-1}\right)$} & $\begin{array}{l}24.51 \\
12.04-49.71\end{array}$ & $\begin{array}{l}21.70 \\
10.84-36.23\end{array}$ & $\begin{array}{l}22.79 \\
13.67-39.40\end{array}$ \\
\hline \multicolumn{2}{|c|}{$\mathrm{Cl}\left(\mathrm{mg} \cdot \mathrm{dm}^{-3}\right)$} & $\begin{array}{l}7.54 \\
0.50-42.38\end{array}$ & $\begin{array}{l}5.43 \\
0.34-31.94\end{array}$ & $\begin{array}{l}5.18 \\
0.31-32.68\end{array}$ \\
\hline \multicolumn{2}{|c|}{$\mathrm{Na}^{+}\left(\mathrm{mg} \cdot \mathrm{dm}^{-3}\right)$} & $\begin{array}{l}5.11 \\
0.00-29.18\end{array}$ & $\begin{array}{l}3.27 \\
0.00-34.54\end{array}$ & $\begin{array}{l}2.58 \\
0.00-21.51\end{array}$ \\
\hline \multicolumn{2}{|c|}{$\mathrm{K}^{+}\left(\mathrm{mg} \cdot \mathrm{dm}^{-3}\right)$} & $\begin{array}{l}15.73^{\mathrm{a}, \mathrm{b}} \\
5.12-43.51\end{array}$ & $\begin{array}{l}12.62^{\mathrm{a}} \\
1.90-39.35\end{array}$ & $\begin{array}{l}10.90^{\mathrm{b}} \\
2.97-34.56\end{array}$ \\
\hline \multicolumn{2}{|c|}{$\mathrm{Ca}^{2+}\left(\mathrm{mg} \cdot \mathrm{dm}^{-3}\right)$} & $\begin{array}{l}24.69 \\
6.56-102.45\end{array}$ & $\begin{array}{l}22.21 \\
5.47-37.77\end{array}$ & $\begin{array}{l}24.85 \\
9.19-49.85\end{array}$ \\
\hline \multicolumn{2}{|c|}{$\mathrm{Mg}^{2+}\left(\mathrm{mg} \cdot \mathrm{dm}^{-3}\right)$} & $\begin{array}{l}3.53 \\
1.09-19.38\end{array}$ & $\begin{array}{l}3.16 \\
0.91-8.68\end{array}$ & $\begin{array}{l}3.91 \\
1.02-9.89\end{array}$ \\
\hline \multicolumn{2}{|c|}{ "Z" coefficient } & $\begin{array}{l}0.152 \\
0.000-0.761\end{array}$ & $\begin{array}{l}0.103 \\
0.000-0.862\end{array}$ & $\begin{array}{l}0.078 \\
0.000-0.575\end{array}$ \\
\hline
\end{tabular}

a. Significantly different pairs with $\mathrm{P}<0.05$, SPR - soil penetration resistance, TOC - total organic carbon, EC-electrical conductivity, EH hydrolytic acidity, TEB - total exchangeable bases, CEC - cation exchange capacity, "Z" coefficient-salinity index "Z".

exhibited values in the range of $1.1-24.0 \%$ (Table 1) and were significantly lower in the Lisia Góra reserve. In investigations of root transpiration and moisture changes in forest areas used for recreation, Komatsu et al. (2007) indicated greater moisture in a habitat that was visited less frequently. de Gouvenain (1995) also found that areas with low tourism pressure were characterised by higher soil moisture. Similar changes in moisture were found by Yüksek et al. (2010) in their investigation of the impact of trampling in protected areas of Turkey. Regardless of the period, there was a statistically significant increase in the value along the distance from the direct sites of tourist interference (Table 2). In the study of areas with different tourism pressures, Zhevelev and Sarah (2008) noticed the same trend toward higher moisture in areas overgrown by natural vegetation. These results were not confirmed in publications of Kissling et al. (2009), who found no differences in the short-term and long-term impact of tourism on soil moisture in distance of $1 \mathrm{~m}$.

The actual acidity ( $\mathrm{pH}$ in water) of the investigated soils ranged from 3.85 to 7.82 and $\mathrm{pH}$ in $\mathrm{KCl}$ was between 3.08-7.05 (Table 1, 2) which indicates that these were mostly acidic soils. Only two places were characterised by neutral reaction: trails under the bridge and near the golf pool. The research sites in the Wisłok Valley were characterised by significantly higher $\mathrm{pH}$ values from in the Lisia Góra reserve sites (Table 1). Similarly, as shown by Sikorski et al. (2013), soil reaction changed significantly in the objects with pedestrian access. It was found in the research conducted by Zdanowicz and Skłodowski (2013) that the $\mathrm{pH}$ values changed with the distance from the trail as well. The soil near the trail was less acidic than that at longer distances, which confirms the slight trend towards changes in the $\mathrm{pH}$ and hydrolytic acidity of the examined soils in the Lisia Góra reserve and Wisłok Valley with significantly less acidic reaction at the distance of $1 \mathrm{~m}$ (Table 2).

The electrical conductivity was in the range of 37.80-534.00 $\mu \mathrm{S} \cdot \mathrm{cm}^{-1}$ (Table 1, 2). There were no statistically significant differences between the distances and sites, but the sites in the Wisłok Valley had higher values of EC (Table 1,2). As shown by Sikorski et al. (2013), salinity changed significantly with a significant impact of tourist pressure in forest park areas, leading to changes in vegetation caused by elimination of some species.

The organic carbon content was in the range of $5.23-53.51 \mathrm{~g} \cdot \mathrm{kg}^{-1}$, with the average content of 27.91 $\mathrm{g} \cdot \mathrm{kg}^{-1}$. There were no clear trends and significant differences between the distances, which indicated absence of a correlation with the changes in soil compaction (Table 1, 2).

There were no statistically significantly differences in the sum of base cations between the distances (with a slightly decreasing trend along the distance). The sites located in the reserve, had a significantly lower sum of base cations, but these were habitats with potentially high acidification and greater values of hydrolytic acidity. These results are reflected in the cation exchange capacity of the soils (Table 1,2 ). 
The salinity index $\mathrm{Z}$ calculated on the basis of ions contained in the soil solution was significantly lowest in the reserve, as well as the content of chloride ions. This trend may be related to the differences in the use of salt to snow removal in the winter, which is prohibited in protected areas (Table 1,2).

Based on the correlation analysis, statistically significant positive Pearson correlation coefficients were found between the actual, exchange and hydrolytic acidity, EC, and soil penetration resistance only in the autumn period, while negative correlations were reported for the sum of base cations (Table 3 ). Kissling et al. (2009) demonstrated that long-term tourism induced changes in the soil reaction in the form of acidification in sites with greater tourism pressure. Andrés-Abellán et al. (2005) showed an increase in the $\mathrm{pH}$ value with the intensity of tourist traffic.

TABLE 3. Pearson correlation coefficients between soil properties

\begin{tabular}{|c|c|c|c|}
\hline \multirow[t]{2}{*}{ Parameter } & & \multicolumn{2}{|c|}{$\mathrm{SPR}\left(\mathrm{kg} \cdot \mathrm{cm}^{-2}\right)$} \\
\hline & & spring & autumn \\
\hline \multirow[t]{2}{*}{ Moisture (\%) } & spring & $\mathrm{ns}$ & - \\
\hline & autumn & - & ns \\
\hline $\begin{array}{l}\text { Fraction }<0.002 \mathrm{~mm} \\
(\%)\end{array}$ & spring & ns & ns \\
\hline $\mathrm{pH} \mathrm{H} \mathrm{H}_{2} \mathrm{O}$ & & $\mathrm{ns}$ & $-0.23 *$ \\
\hline $\mathrm{pH} \mathrm{KCl}$ & & $\mathrm{ns}$ & $-0.28 *$ \\
\hline TOC $\left(\mathrm{g} \cdot \mathrm{kg}^{-1}\right)$ & & $-0.30^{*}$ & ns \\
\hline $\mathrm{EC}\left(\mu \mathrm{S} \cdot \mathrm{cm}^{-1}\right)$ & & ns & $-0.19 *$ \\
\hline $\mathrm{EH}\left(\mathrm{cmol}(+) \cdot \mathrm{kg}^{-1}\right)$ & & $\mathrm{ns}$ & $0.37 *$ \\
\hline $\mathrm{TEB}\left(\operatorname{cmol}(+) \cdot \mathrm{kg}^{-1}\right)$ & & $\mathrm{ns}$ & $-0.20 *$ \\
\hline$\overline{\mathrm{CEC}\left(\mathrm{cmol}(+) \cdot \mathrm{kg}^{-1}\right)}$ & & ns & ns \\
\hline $\mathrm{Cl}^{-}\left(\mathrm{mg} \cdot \mathrm{dm}^{-3}\right)$ & & $\mathrm{ns}$ & ns \\
\hline $\mathrm{Na}^{+}\left(\mathrm{mg} \cdot \mathrm{dm}^{-3}\right)$ & & $\mathrm{ns}$ & ns \\
\hline $\mathrm{K}^{+}\left(\mathrm{mg} \cdot \mathrm{dm}^{-3}\right)$ & & $\mathrm{ns}$ & ns \\
\hline $\mathrm{Ca}^{2+}\left(\mathrm{mg} \cdot \mathrm{dm}^{-3}\right)$ & & $\mathrm{ns}$ & $-0.42 *$ \\
\hline $\mathrm{Mg}^{2+}\left(\mathrm{mg} \cdot \mathrm{dm}^{-3}\right)$ & & $\mathrm{ns}$ & $-0.34 *$ \\
\hline "Z" coefficient & & $0.22 *$ & $0.21 *$ \\
\hline
\end{tabular}

*significant correlations for $\mathrm{p}<0.05$; ns - not significant, SPR - soil penetration resistance, TOC - total organic carbon, EC - electrical conductivity, EH - hydrolytic acidity, TEB - total exchangeable bases, CEC - cation exchange capacity, "Z" coefficient-salinity index " $Z$ ".

\section{CONCLUSIONS}

1. The average values of soil penetration resistance were significantly higher directly on the trails regardless of the research period and decreased at the distance of $1 \mathrm{~m}$ from the trail.

2. There was an increase in the soil penetration resistance in autumn caused by intense holiday season tourist traffic.
3. Based on the results of Kruskal-Wallis test, it can be concluded that the soil penetration resistance is significantly higher in the Lisia Góra Nature Reserve site.

4. Soil moisture was significantly lower at places with intensive trampling like the playground and the reserve bench area, though only in autumn. There were no significant changes between distances.

5. There were no significant changes in soil properties between distances. According the Kruskal-Wallis test, most site soil properties change significantly and places in the Lisia Góra Nature Reserve were characterized by more acidic reaction and smaller sorption properties.

6. The „Z" coefficient was significantly lower for the trails from the nature reserve with lower anthropopressure in comparison with the objects from the Wisłok Valley where tourism pressure is higher.

\section{REFERENCES}

Andrés-Abellán M., Benayas Del Álamo J., Landete-Castillejos T., López-Serrano F. R., García-Morote F.A., del Cerro-Barja A., 2005. Impacts of visitors on soil and vegetation of the recreational area "Nacimiento del Río Mundo" (Castilla-la Mancha, Spain). Environmental Monitoring Assessment 101: 55-67.

Bajda-Gołębiewska M., 2011. Szlaki turystyczne na obszarach chronionych. Economy and Management 1: 57-69.

Breś W., 2008. Czynniki antopopresji powodujące zamieranie drzew w krajobrazie miejskim. Nauka Przyroda Technologie, T. 2, z. 4: 1-8.

Ćwik A., 2009. The role of Wisłok Valley in Rzeszów in the opinion of the town's citizens. Architektura krajobrazu 24: 65-70.

Ćwik A., Ćwik Ł., 2011. The anthropopressure in the Lisia Góra Nature Reserve in Rzeszów. Chrońmy przyrodę ojczystą T67, nr 5: 441-448.

De Gouvenain C.R., 1996. Indirect impacts of soil trampling on tree growth and plant succession in the north Cascade Mountains of Washington. Biological Conservation 75: 279-287.

Ewertowski M., Tomczyk A., 2007. Ocena stanu środowiska geograficznego szlaków turystycznych - wykorzystanie GIS do integracji i analizy danych terenowych i kartograficznych. Przegląd Geograficzny 79, 2: 271-295.

Jim C.Y., 1998. Soil characteristics and management in an Urban Park in Hong Kong. Environmental Management vol. 22, No. 5: 683-695.

Kissling M., Hegetschweiler T.K., Rusterholz H-P., Baur B., 2009. Short-term and long-term effects of human trampling on aboveground vegetation, soil density, soil organic matter and soil microbial processes in suburban beech forests. Applied Soil Ecology 42: 303-314.

Komatsu H., Katayama A., Hirose S., Kume A., Higashi N., Ogawa S., Otsuki K., 2007. Reduction in soil water availability and tree transpiration in a forest with pedestrian trampling. Agricultural and Forest Meteorology 146: 107-114. 
Littlemore J., Barker S., 2001. The ecological response of forest ground flora and soils to experimental trampling in British urban woodlands. Urban Ecosystems 5: 257-276.

Machnik A., 2010. Wybrane modele ekoturystyki na obszarach przyrodniczo cennych w Polsce. Geoturystyka 21(2): 19-26.

Mika M., 2000. Turystyka jako czynnik przemian środowiska przyrodniczego - stan badań. Prace geograficzne z. 106: 7398.

Millward A.A., Paudel K., Briggs S.E., 2011. Naturalization as a strategy for improving soil physical characteristics in a forested urban park. Urban Ecosystem 14: 261-278.

Mocek A., Drzymała S., Maszner P., 1997. Geneza, analiza i klasyfikacja gleb. Wydaw. Dydaktyczne AR w Poznaniu. Poznań: 202-274.

Mroczek J.R., Kostecka J., 2008. Rola Zalewu Rzeszowskiego w zrównoważonym rozwoju miasta Rzeszowa. Inżynieria Ekologiczna nr 20: 50-56.

Ostrowska A., Porębska G., Borzyszkowski J., Król H., Gawliński S., 2001. Właściwości gleb leśnych i metody ich oznaczania. Dział Wydawnictw IOŚ, Warszawa: 108 pp.

Pickering C.M., Hill W., 2007. Impacts of recreation and tourism on plant biodiversity and vegetation in protected areas in Australia. Journal of Environmental Management 85: 791800.

PN-ISO 10390: 1997. Jakość gleby - Oznaczanie pH.

PN-ISO 11265+AC1: 1997. Jakość gleby - Oznaczanie przewodności elektrycznej właściwej.
Polskie Towarzystwo Gleboznawcze, 2009. Klasyfikacja uziarnienia gleb i utworów mineralnych - PTG 2008. Roczniki Gleboznawcze - Soil Science Annual 60(2): 5-16.

Sikorski P., Szumacher I., Sikorska D., Kozak M., Wierzba M., 2013. Effects of visitor pressure on understory vegetation in Warsaw forested parks (Poland). Environtal Monitoring Assessment 185: 5823-5836.

Szpilka D., Ziółkowski R., 2010. Zagospodarowanie turystyczne obszarów chronionych województwa podlaskiego. Economy and Management 1: 52-73.

Talbot L.M., Turtona S.M., Graham A.W., 2003. Trampling resistance of tropical rainforest soils and vegetation in the wet tropics of north east Australia. Journal of Environmental Management 69: 63-69.

Yüksek T., Kurdoğlu O., Yüksek F., 2010. The effects of land use changes and management types on surface soil properties in Kafkasör protected area in Artvin, Turkey. Land Degradation Development 21: 582-590.

Zdanowicz E., Skłodowski J., 2013. Ocena zmian w środowisku wokół szlaków rekreacyjnych na przykładzie rezerwatu Las Bielański w Warszawie. Studia i Materiały CEPL w Rogowie 37: 348-355.

Zhevelev H., Pariente S., 2008. The effect of visitors' pressure on the spatial variability of sandy soil in urban park in the Tel Aviv. Environmental Monitoring Assessment 142: 35-46.

Received: June 12, 2016

Accepted: June 5, 2017

Associated editor: D. Częińska-Kamińska

\section{Wpływ rekreacji i turystyki na wybrane wlaściwości gleb na obszarze rezerwatu Lisia Góra w Rzeszowie}

Streszczenie: Celem badań było określenie zmian właściwości gleb, powstałych przez intensywną rekreację i turystykę w obszarach chronionych, usytuowanych w granicach miasta Rzeszów (południowo-wschodnia Polska). Prace badawcze były prowadzone w dwóch obiektach: (1) na obszarze chronionym rezerwatu Lisia Góra i (2) wzdłuż korytarza rzeki Wisłok. Uwzględniono miejsca o różnym natężeniu antropopresji. W sezonie wiosennym i jesiennym oznaczono odporność na ściskanie i wilgotność gleby. W materiale glebowym pobranym wiosną oznaczono: skład granulometryczny, $\mathrm{pH}$, węgiel organiczny, EC w roztworze gleba:woda 1:10. W tym samym ekstrakcie oznaczono zawartość jonów $\left(\mathrm{Ca}^{2+}, \mathrm{Mg}^{2+}, \mathrm{K}^{+}, \mathrm{Na}^{+}\right)$za pomocą chromatografu jonowego. Odnotowano występowanie istotnej korelacji (określonej przez współczynnik Pearson) pomiędzy kwasowością gleby, EC, sumą kationów zasadowych, jonami wapnia i magnezu a odpornością na ściskanie gleby. Stwierdzono istotne różnice w zbiciu gleby w zależności od obiektu (większe na terenie korytarza Wisłoka) oraz odległości od granicy miejsc intensywnie użytkowanych (zmniejszanie się ze wzrostem odległości). Współczynnik „Z” (stosunek jonów sodu do sumy wapnia i magnezu z roztworu wodnego w stosunku gleba-woda 1:10) był istotnie niższy dla miejsc znajdujących się na terenie rezerwatu.

Słowa kluczowe: odporność na ściskanie, przewodność elektryczna, współczynnik Z, turystyka, obszar chroniony 